\title{
Modern Control and Diagnostic System of Traction Vehicle with Hybrid Drive System
}

\author{
Zygnunt Szymański \\ Silesian University of Technology Gliwice, Str Akademicka 244-100, Gliwice, Poland
}

\begin{abstract}
In paper it introduced a review of modern traction vehicle drive system with induction motor drive system (PMSM with single or dual rotor drive system) or BLDC motor with different configuration of magnetic circuits. For particular part of drive system proposed a quasi intelligent control system version smart control enables multi criteria predictive control of vehicle work. In the paper presented also a selected diagnostic procedure, enables monitoring exploitation parameters, and prediction of probable failure state. For different vehicle work state realized a simulation models and crash test of exploitations failure models.
\end{abstract}

Key words: Smart control, diagnostic model, hybrid vehicle.

\section{Introduction}

The mass-development of internal-combustion transport creates real threats of natural environmental pollution, generates excessive noise, twitches and vibrations, and can be also a source of climatic changes. One variant a limitation of influences is application of new type drive system: electric or hybrid (electric motor, electric motor and internal-combustion engine, drive composed with internal-combustion engine and mechanical energy container). Hybrid vehicle is an economically and technically most simple solution. Augmenting of classical internal-combustion drive system, electric motor diminishes fuel consumption, reduces issue of impurities, keeping at this dynamics and comfort of the vehicle drive. In the paper it introduced a review of modern wheel vehicle drive system with induction motors drive system (PMSM permanent magnet synchronous induction motor with single or dual rotor) or BLDC motor with different configuration of magnetic circuit. For hybrid drive system proposed a quasi intelligent control system version smart control, enables multicriterial, predictive control of vehicle

Corresponding author: Szymanski Zygnunt, D.Sc., research fields: modern diagnostic and control method of drive system, smart predictive smart system. work system. In the paper presented also a selected diagnostic procedure enables monitoring exploitations parameters, and prediction of probable failure state. For different vehicle work state realized a simulation models and crash test of exploitations failure models. In analysis of exploitation dynamic states used computer program: PSIM, Portunus, and Simulation X. Results of computer calculations one verified with laboratory-measurement results, put in suitable corrections to simulation models.

\section{Review of Construction Solution a Drive System}

In hybrid vehicles construction with rows drive system is used a combustion engine drive generator, creating current aggregate to battery charge of batteries, and to power supply of supply inverter. The electric traction motor is supply with batteries by the supply inverter block, he driving by drive carriage system, driving axles of vehicle. Vehicles with parallels drive to driving units: combustion engine, electric motor and drive parallel driving axles of the vehicle. During applying of the vehicle brake, electric motor runs into the range of generator work and supercharges batteries. Hybrid drives found a wide use in vehicles: military (vehicle AHED $8 \times 8$ ), municipal 
(vehicles Solaris Urbino Hybrid), communication, and as special vehicles traction (MONTRAKS), used in repair works of the trolley wires. Fig. 1 introduced the visual schema a drive system of hybrid vehicle Solaris
Urbino Hybrid [1].

Fig. 2 shows a 2D drawing of the FCAFPM (field controlled axial flux permanent magnet) motor.

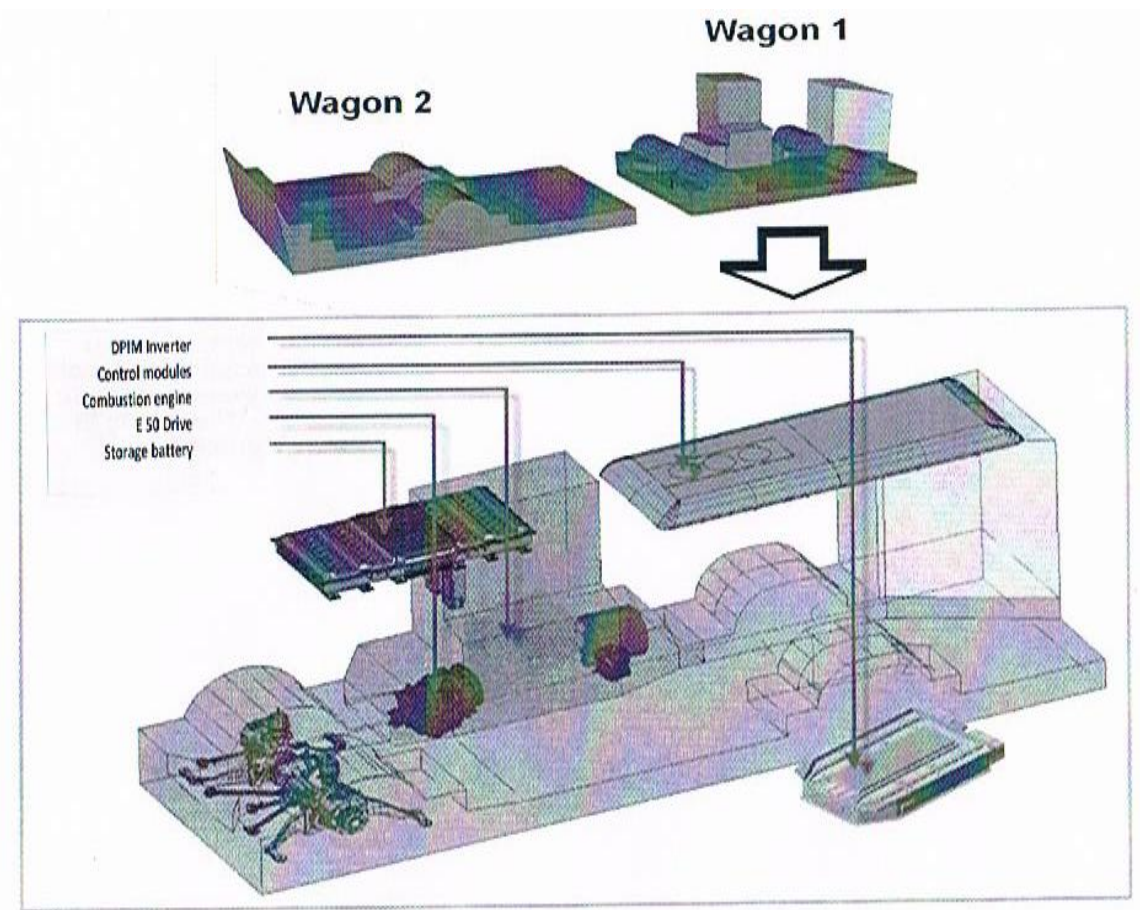

Fig. 1 The visual schema of the drive system of vehicle type Solaris Urbino Hybrid [2].

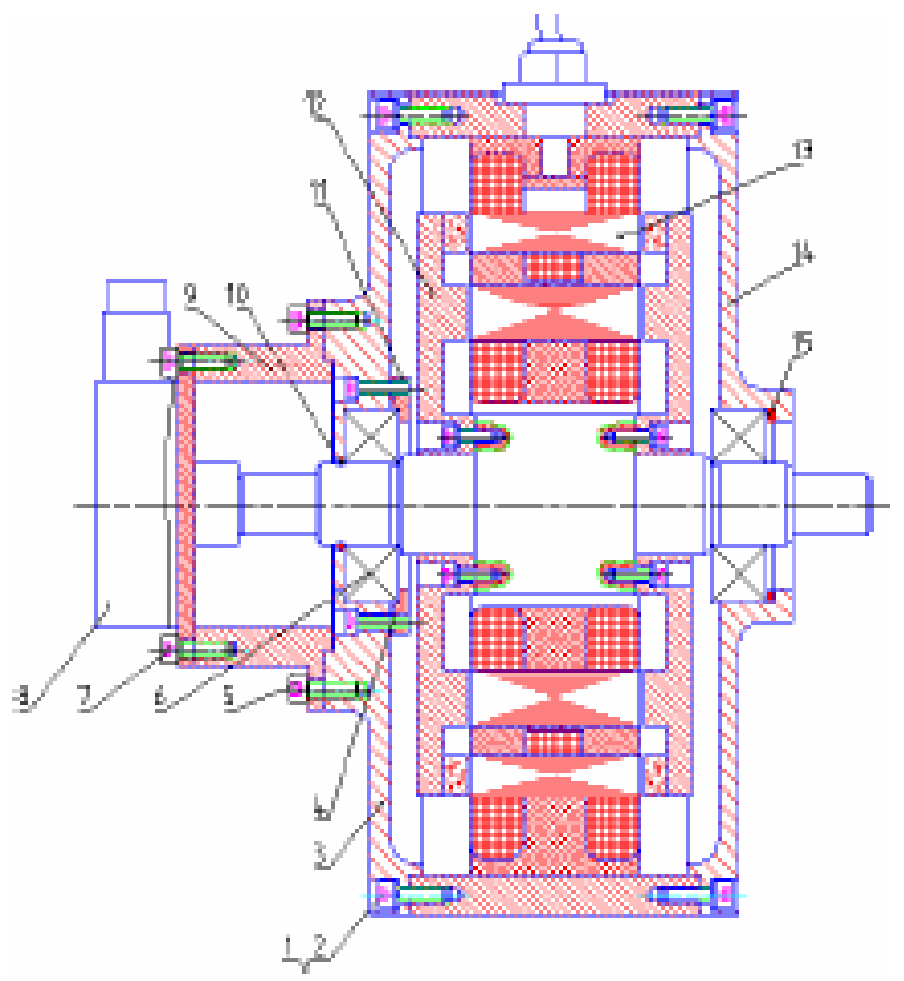

1. Bul

2 Dasher

3. Firwinl Fad

$\mathrm{Br} 1$

4. Bul

S. EN

i. Bearify

7. BdI

B. Rorthith

9. Coule fis 5 버

10. Heirie

heside Rige

11. Hewring

Bufle

12. Buns

intwity

13. Sthin

14. Bask Fan

Bel

15. Bipk Gubet

Fig. 2 The visual scheme of FCAFOPM motor [2]. 
The stator structure is formed by two-strip wound or tape wound stator rings, circumferentially wound DC field winding and two sets of 3-phase AC windings. The stator core is divided into two sections in order to place the DC field winding in between, which makes it possible to vary the net air gap flux. The stator core also has slots to accommodate the two sets of 3 phase $\mathrm{AC}$ windings which are connected in parallel. The rotor is divided into two parts. The upper permanent magnets of the left rotor, which are mounted on every other pole, are magnetized as N-poles and aligned with the upper permanent magnets of the right rotor which are magnetized as S-poles. The lower section of the pole is a non-magnet pole of iron core. Similarly, the magnets in the lower sections of the poles are magnetized as S-poles which are again located on every other pole and aligned with the N-pole side magnets.

PEVs (Plug in electric vehicles) are propelled by an electric motor powered by a utility charged battery source. The recent studies show that the overall efficiency and reliability of the electric propulsion system can be further improved by replacing the MD (mechanical differential) with the ED (electrical differential) for facilitating the turns around curvatures $[1,3]$. Moreover, the use of ED results in reduced driveline parts, weight, and simplified mechanical layout. Generally, multiple propulsion motors are needed to achieve the ED. This use of multiple propulsion motors causes increase in under spring mass and system complexity due to the increased power and signal electronics. The control of two individual ac motors with a five-leg VSI (voltage source inverter), can be employed for ED control of PEV. However the five-leg VSI increases switch count as compared to the three-leg VSI. The dual-rotor motor consists of two independent rotors and a single stator have reported the physical design and analysis of dual-rotor induction motor and dual-rotor PM (permanent magnet) motor. Energy efficiency predominantly is vital in PEVs as it is directly related to the travel distance per charge, battery size, and vehicle cost. PMSM has the merits of higher power density, high power factor, smaller size and maintenance-free operation. The dual-rotor PMSM combines the advantages of reduced copper losses and leakage flux, higher utilization of the stator winding, and inherent ED with that of the PMSM. Szymański [1] and Chee Shen Lim et al. [3] report three methods for the vector control of dual-rotor PMSM. Method-I implements the control of sum of two rotor speeds instead of controlling the individual speeds. Method-II, involves the control of individual speed errors to determine the average value of the reference $q$-axis current [4]. Furthermore, method-II controls the average torque without considering the angular difference between the two reference $q$-axis currents. With these two methods, the inner and outer rotor speeds will be equal provided that the machine parameters are exactly matched.

The difference in rotor inertias due to the different inner and outer rotor diameters will affect the vehicle control performance. These methods necessitate MD as the two rotor speeds are controlled at equal reference speed reports the vector control of dual-rotor PMSM where the two rotors speeds are controlled at a common reference speed to eliminate the coupling effect. Method III proposes the detailed four axes $d-q$ model of dual-rotor PMSM, where the PMs are embedded in the rotor structure, thereby making it an interior PMSM. The angular displacement between the $d-q$ axes of the outer and inner rotor is determined with the respective $d$-axis fluxes. Additionally, the proposed PEV system is examined for additional three driving cycles to test stability and robustness of the control algorithm over the varying speed-torque profiles. The outer rotor has PMs at the outside surface of the outer air gap with the flux directed inward/outward, and the inner rotor has PMs at the inside surface of the inner air gap with the flux directed outward/inward [3]. The inner and outer surfaces of stator are uniformly slotted and mounted 
with a torroidal winding, which allows for the reduction in length of winding. Hence, the leakage inductance and resistance of the stator windings is less as compared to conventional electric machines. This results in reduced copper losses and leakage, leading to higher efficiency. In dual-rotor PMSM, the outer and inner rotors are connected together to be one end disc and the stator is fixed to a frame at the other end. When the three-phase symmetrical currents are supplied to stator windings, the opposite rotating magnetic fields are developed at the two sides of the stator $[1,3]$. The back-emf is induced by the flux travelling circumferentially in the stator core and as the directions of both current and flux for the inner air gap are opposite to that for the outer air gap, two tangential forces produced in the inner and outer air gaps by interaction of the current commanded in the winding coils with the flux generated by the magnets have the same orientation [5]. The two rotating fields separately interact with respective rotor flux to develop the opposite electromagnetic torques. Furthermore, this machine has the merits of high overload capability, low cogging torque, low material costs, higher torque density and efficiency over induction and PM machines. Fig. 3 shows the schematic diagram of dual rotor PMSM based propulsion system for driving a four-wheeler PEV. The propulsion system consists of a dual-rotor PMSM, VSI, bidirectional dc-dc converter, lithium-ion battery pack, necessary sensors and digital controller. The inner and outer rotors of the dual-rotor PMSM propel the right and left rear wheels, respectively. The lithium-ion battery pack supplies the VSI fed dual-rotor PMSM drive, through the bidirectional dc-dc converter. The digital controller implements the algorithms for the control of stator currents and the input/output voltages of dc-dc converter.

As the inner and outer rotor rotate in opposite directions, a reverse gear is included for one of the wheels (here, for inner rotor driven right rear wheel), to ensure that the torque is applied to propel the PEV in a common direction. This gear reverses the direction of force being applied at the wheel. Additionally, fixed gears are employed for both the rear wheels as the vehicle speed is not same as the rotor speed. When the proposed PEV system operates in acceleration and cruising modes, the dual rotor PMSM is in the motoring mode and the two rotors develop the commanded torque to propel the individual wheels thereby driving the PEV. In the braking mode, the two rotors are driven by the respective wheels and the dual-rotor PMSM operates as a generator facilitating the charging of the battery pack by utilizing the kinetic energy of the PEV, thus providing regenerative braking. During ED control, the two rotors rotate at different speeds to allow for turning around the curvatures. The details of the battery model and the bidirectional dc-dc converter are discussed in the following sub-sections. Fig. 4 shows four wheeler PEV with dual rotor PMSM [3].

\section{Multicriterial Predictive Control System}

An alternative solution is applied in a sub optimal control of hoisting-machine. This multi-level control system is applied in a multi criteria, high size controller. The sub optimal control enables the realization of adaptive algorithms of the control with the regard of occurrences anticipatory their pronouncement. The predictive control assuring optimum-control with the composition of hoisting machine drives at established functions of aim and multi criteria, determining the proper machine work in

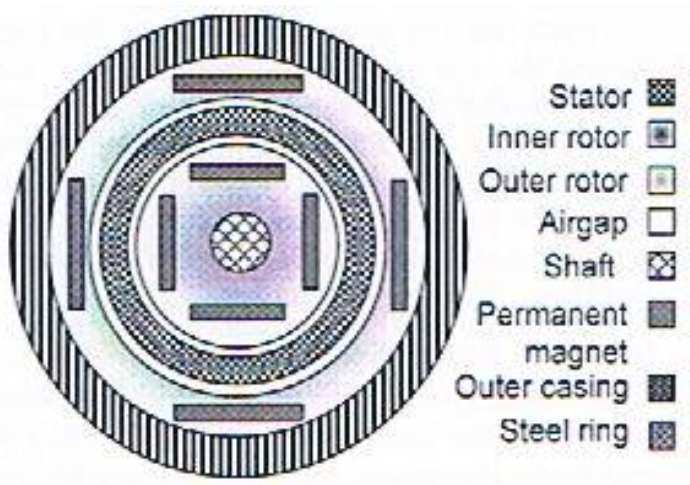

Fig. 3 Schematic diagram of dual rotor PMSM [3]. 


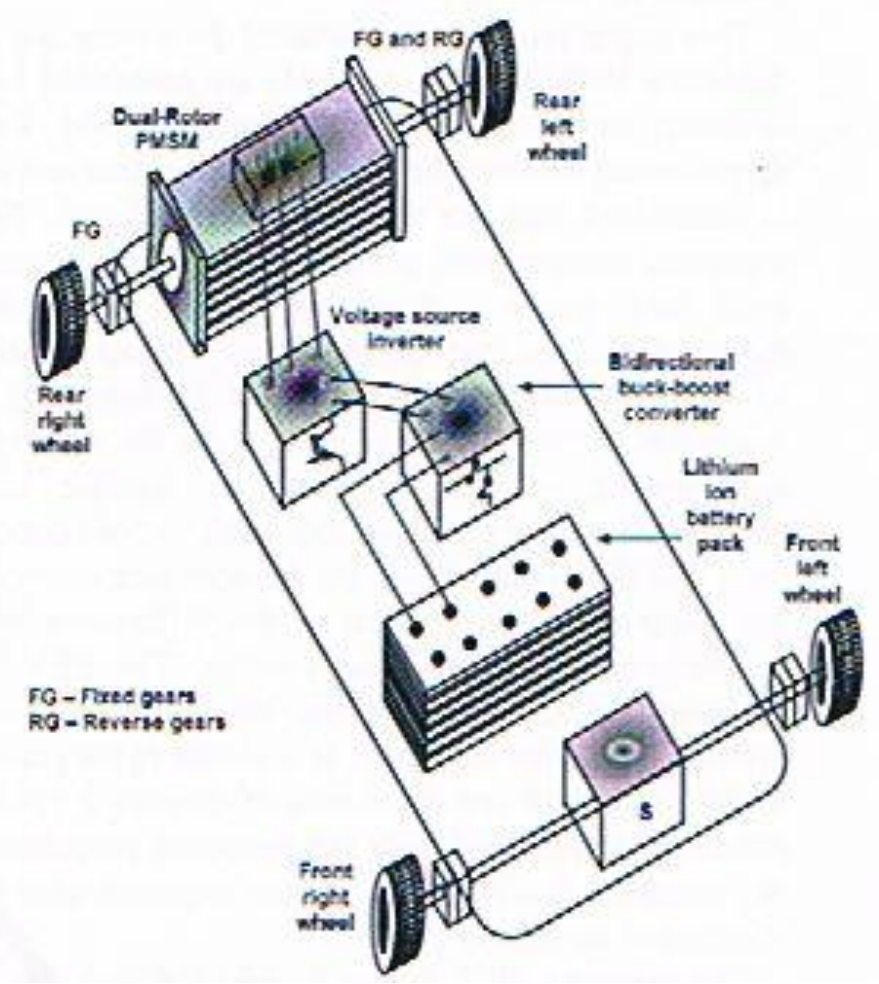

Fig. 4 Four wheeler PEV with dual rotor PMSM $[1,3]$.

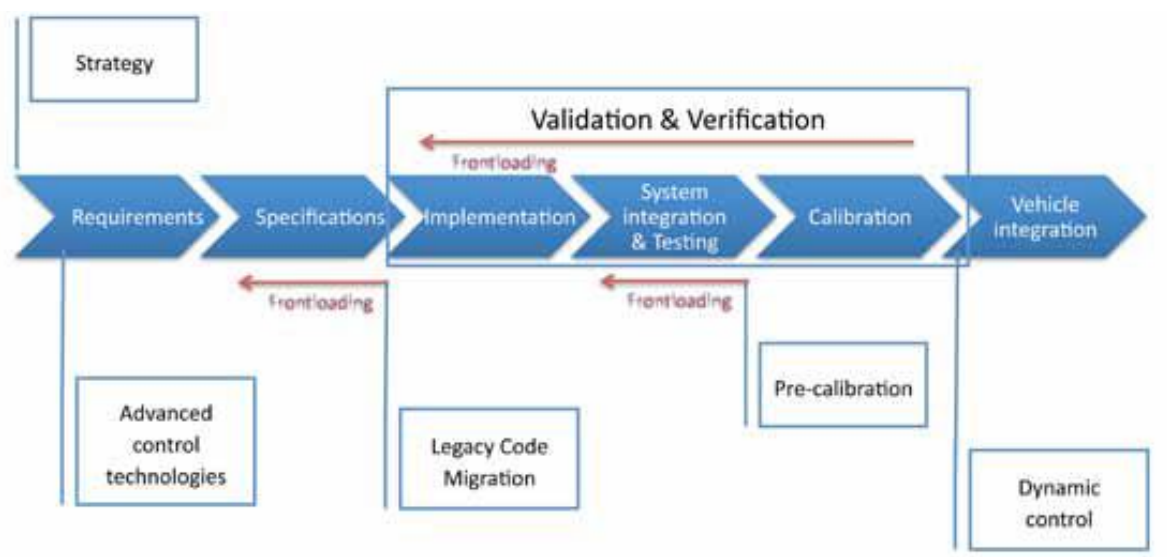

Fig. 5 Block scheme of design strategy a smart control vehicle [6].

compliance with realized technical process. Predictive control: MPC (model predictive control) or RHC (receding horizon control) is methods of control a non-linear dynamic system, consisting in cyclical resolution of properly formulating assignments of optimum-(ZSO) control. The first part of solution (functions master) is given on input of regulation object, then solve a differential equations systems, describing dynamic model of the object, obtained results of calculations are compared with the real state, then all procedures are repeated again for new established state of object. Mathematical model of predictive control system describes equations system (1):

$$
\begin{aligned}
& x(t)=f[x(t), u(t)] x(t) \in X \subset R^{n} \\
& u(t) \in R \in U \quad u_{\min } \leq u \leq u_{i \max } \\
& u_{\min }<0, \quad u_{i_{\max }} \geq 0
\end{aligned}
$$


$\mathrm{x}(\mathrm{t}), \mathrm{u}(\mathrm{t})$ - object control functions;

$\mathrm{f}(\mathrm{x}, \mathrm{u})$ - functions realizing algorithms of the optimum-control.

Eq. (1) must realize the Lipschitz condition: $f(0,0)$ $=0$. Quality coefficient of the object control $J$ we can calculate from Eq. (2):

$$
J_{i}\left(u^{i}, T_{i}, x\left(t_{i}\right)\right)=\int_{t_{i}}^{t_{i}+T_{i}} L\left(x^{i}, u^{i}\right) d t+q\left(x^{i}\left(t_{i}+T_{i}\right)\right)
$$

where:

$\mathrm{L}(\mathrm{x}, \mathrm{u})$ - total energy of drive system;

$\mathrm{q}(\mathrm{x})$ - individual energy of $i$ element of drive system.

Parameters of optimum-regulator described with the dependence: $\mathrm{u}=\mathrm{k}(\mathrm{x})$, can marked dissolving Hamilton-Jacobi-Bellman equations [4, 6]. Finding of optimum-solution at the predictive control system is largely impossible for composite objects of regulation with non-linear system. At the steady state structure of control system one seeks with gradient methods the minimum of the quality coefficient $\mathrm{Q}(\mathrm{p})$, where $\mathrm{p}$ is a vector of parameters of the control system. The gradient of quality coefficient of the visage complies the numeric integration of equations of state and conjugates equalizations of mining-machine driving system. During realization of optimization calculations generated are procedures of the generation and the reduction. Different manners of generating of procedures are practical: pins, one-nodal, flat and multinomial [6, 7]. The pins generation consists in introduction of additional predictive pulse to the control algorithm, and to calculate an optimum-solution. The one-nodal generation consists in the introduction of new node to matrix of control system. The flat generation consists in the introduction of limitations: of minimum and maximum to procedures of control system. The multinomial generation causes the enlargement about 2 meltings of the polynomial $(n+2)$ describing the process of object control system. After the process generation, it follows the structure change of control circuit, and change of dimension a decision space. The process of research is continued in new space, to next process generation, or to realization of necessary conditions to obtainment of optimum-solution. Procedures of process reduction consist in effacement of limitations of technical realizations of established procedures. The hoisting-machine can be treated as the non-linear object with nonholonomic nodes, described systems of differential non-linear equations $[2,8]$.

\section{Control with application of genetic algorithms} consists in the exchange of the real control system with the system genetic, composite from: cells, tissues, networks of connections of inter cell and superior and local decision units the single cell contains: two microprocessors, transducers $\mathrm{A} / \mathrm{C}$ and $\mathrm{C} / \mathrm{A}$, transmission registers, and operation memory. The cell copies the physically definite fragment of the real control circuit. Describing according to plan dependences happened among each elements of the control circuit and the controller we build the network of connections among each cells (with genes) of the system. It is possible that this model of physical phenomena happened in the real control system. The control system requires application of more microprocessor drivers and is advisable at the control of extensive and complicated processes technical. The application of expert systems in control systems of traction vehicle will assure: the energy-saving manner of the power supply and the control the work of: drive elements and running inspection of exploitive parameters, the diagnostics local and total of elements of traction system, and optimal control with the work of combustion engine.

\section{Smart Control System of Hybrid Traction Vehicle}

The automotive wheel vehicle manufacturers are under pressure to stay technologically ahead and to develop cutting-edge products that are less expensive, according with governmental regulations and industry 
standards, such as ISO 26262, which requires to produce more eco-friendly, safe and smart products. To achieve these goals, car makers are applying mechatronic solutions to develop breakthrough products with brand-differentiating functionalities. Mechatronic solutions leverage electronics and software in advanced control systems to optimize the performance and efficiency of mechanical system.

Mechatronic solutions result in smart systems that not only adapt to human driving patterns, but also to the operating environment and to the state of the vehicle. In addition, they incorporate prognostics and diagnostics to ensure that the vehicle operates well within its performance envelope and any deviation from this performance envelope is managed to ensure the safety of the vehicle occupants. Control system design processes and methods are crucial to developing, deploying and improving high quality system-specific controls in a minimum amount of time. Relying on domain, tool and embedded software experience, LMS has development processes to efficiently deliver high quality control software. LMS' requirements-driven approach offers a one-stop solution to mechatronic system development, resulting in an implementable control system structure that can directly be used for automatic code generation. LMS has wide experience in all phases of the controls development process including requirements definition, systems integration, testing and final calibration. In addition applying in controls development processes and software tools, LMS is also proficient in advanced control techniques, such as MEC (Model Embedded Control) and MPC (Model Predictive Control). LMS' experience includes internal combustion engines, hybrid engines, transmissions, energy storage and chassis applications. To avoid the time-consuming and expensive traditional hardware-based build-test-fix process, LMS' approach enables early decision making and early identification of issues by proceeding with control system development before availability of prototype hardware.

The LMS model-based approach to control strategy development delivers an implementable control system structure that can be directly used to automatically generate code for the target application. LMS helps co procedures develop, deploy and continuously improve an organization specific controls development process. From requirements, system integration and testing to final calibration, LMS leverages hands-on MBSE experience to help its customers concurrently develop the physical system and its associated controller. One of the advantages of using a model-based approach to controller development is the ability to generate embedded software in the form of C-code directly from the models. LMS has developed structured application-specific models and code generation processes that produce high quality control software with minimal development times. LMS engineers utilize domain, tool and embedded software experience while complying with organization-specific standards to set up the modeling and code generation process so that the generated software is $100 \%$ functionally correct. Fig. 6 shows a LMS design of smart control hybrid vehicle. MPC (Model Predictive Control) provides a systematic approach for designing highly complex multivariable control systems. Essentially, the MPC approach optimizes a control objective over a finite horizon in the future, using a system model to predict the system response. A fundamental advantage of MPC is that system limitations are explicitly taken into account in the controller description by adding them as constraints to the optimization problem.

Consequently, the controller can exploit the full system performance. MPC also provides a systematic, streamlined procedure to analyze a model's level of accuracy and complexity, create compact control models, preferably directly from a high-fidelity system model, analyze the performance and computational complexity of the resulting controller 


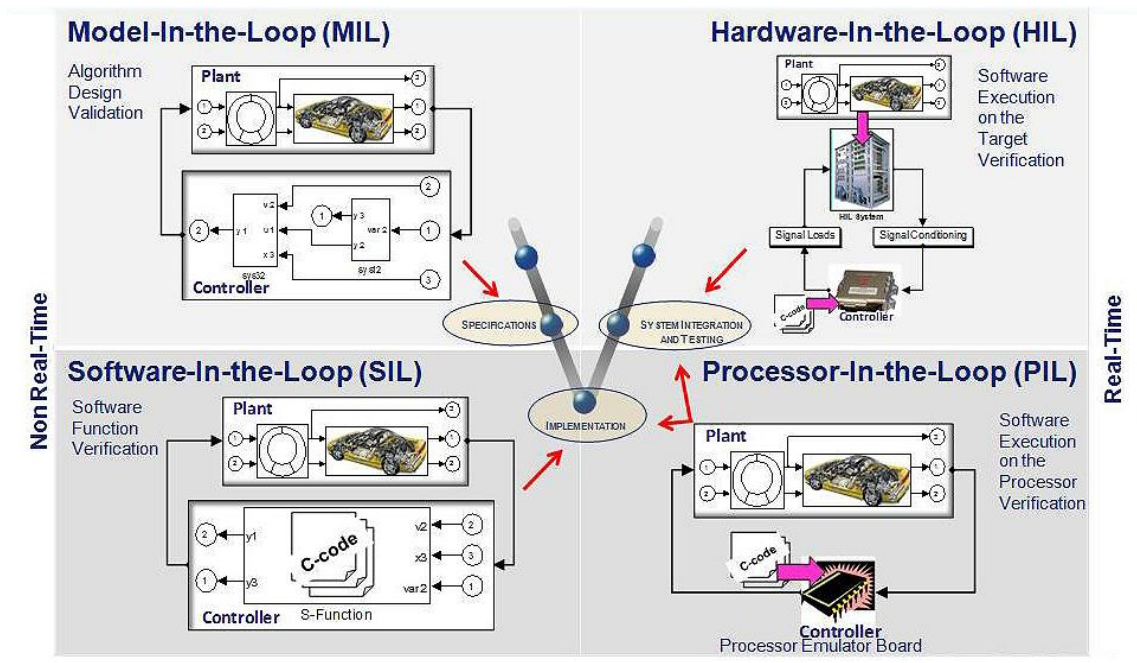

Fig. 6 LMS design of smart control hybrid vehicle.

when simulated in closed-loop with the high fidelity plant model and assess the system performance envelope by adjusting the control objective. MEC (Model-Embedded Control) is a numerical technique that automatically generates the optimal control trajectories for a given plant model. The controller uses a copy of the plant model to evaluate possible control actions, returning the action that achieves the user-specified objective. MEC provides an upper limit of performance by determining that certain systems will never meet the requirements even under ideal control. This approach makes it possible to quickly zero in on the best possible design. By focusing on what the controller should do, and not on how it should do so, MEC also provides more concrete controller requirements and insight into good control strategies, with the advent of new types of engine and transmission actuators and fundamental breakthroughs in reducing losses and waste energy recovery technologies. Modeling is equally essential to develop an optimal control solution for these technologies.

With its vast thermal, hydraulic, electrical and mechanical libraries and components, the performance impact of a new actuator can be evaluated in conjunction with control before committing to hardware prototypes. The use of models earlier in the design process results in more easily controlled physical system designs. This increases the quality and productivity of the design process. LMS Imagine. Fig. 7 shows a screen of computer model a smart design of vehicle. Lab AMESim libraries support the development of both conventional powertrain systems and new technology vehicles such as BEVs (battery electric vehicles), HEVs (hybrid electric vehicles), PHEVs (plug-in hybrid electric vehicles), and FCVs (fuel cell vehicles). Standard drive scenarios for fuel economy and emission prediction are also included. In the paper based also on original version of smart design modules prepared by author in Fig. 8 presented a visual scheme of the fuzzy logic neuro net controller of traction vehicle.

\section{Modern Diagnostic System of Wheel Vehicle}

A conventional system for predicting and evading crash of a vehicle is described first to facilitate an understanding of the present invention. Throughout the following detailed description, similar reference numerals refer to similar element in all figures of the drawing. In the following description, the term "crash" is used in a wider sense that relates to all unexpected traffic accidents. Accidents other than crash include a turnover or fall of a vehicle, with which the phenomenon of "crash" is associated in 


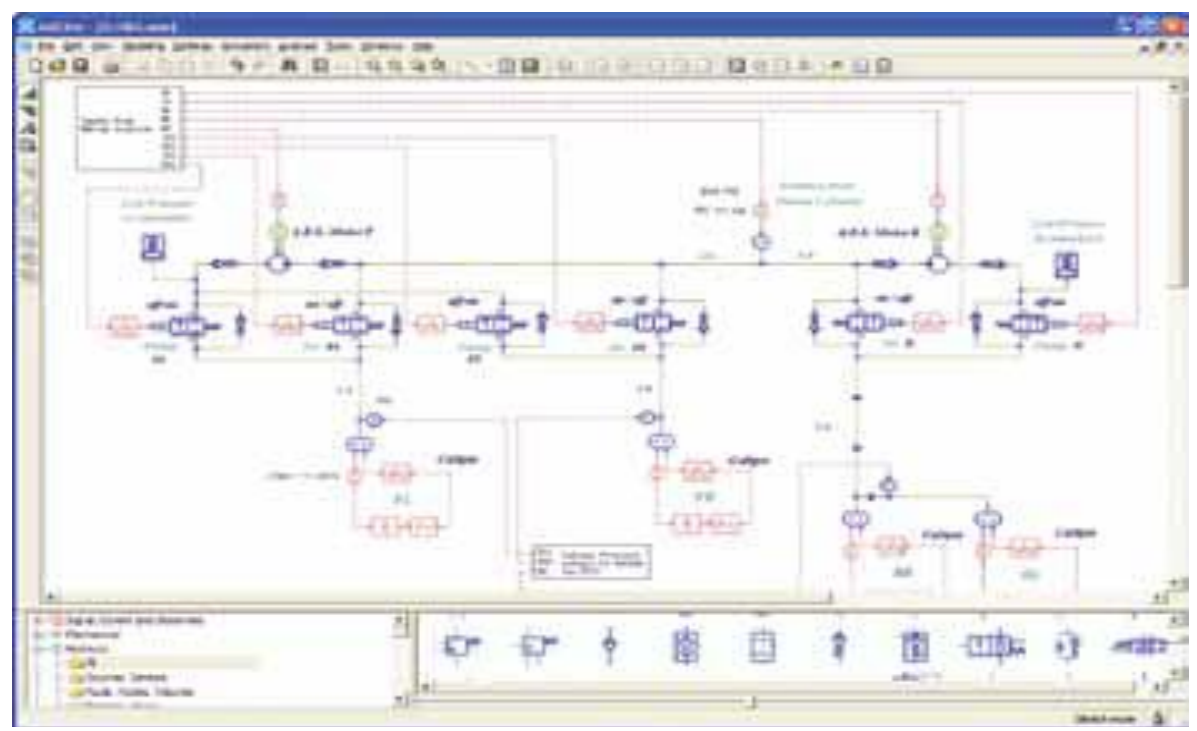

Fig. 7 Screen of computer model a smart design of vehicle [6].

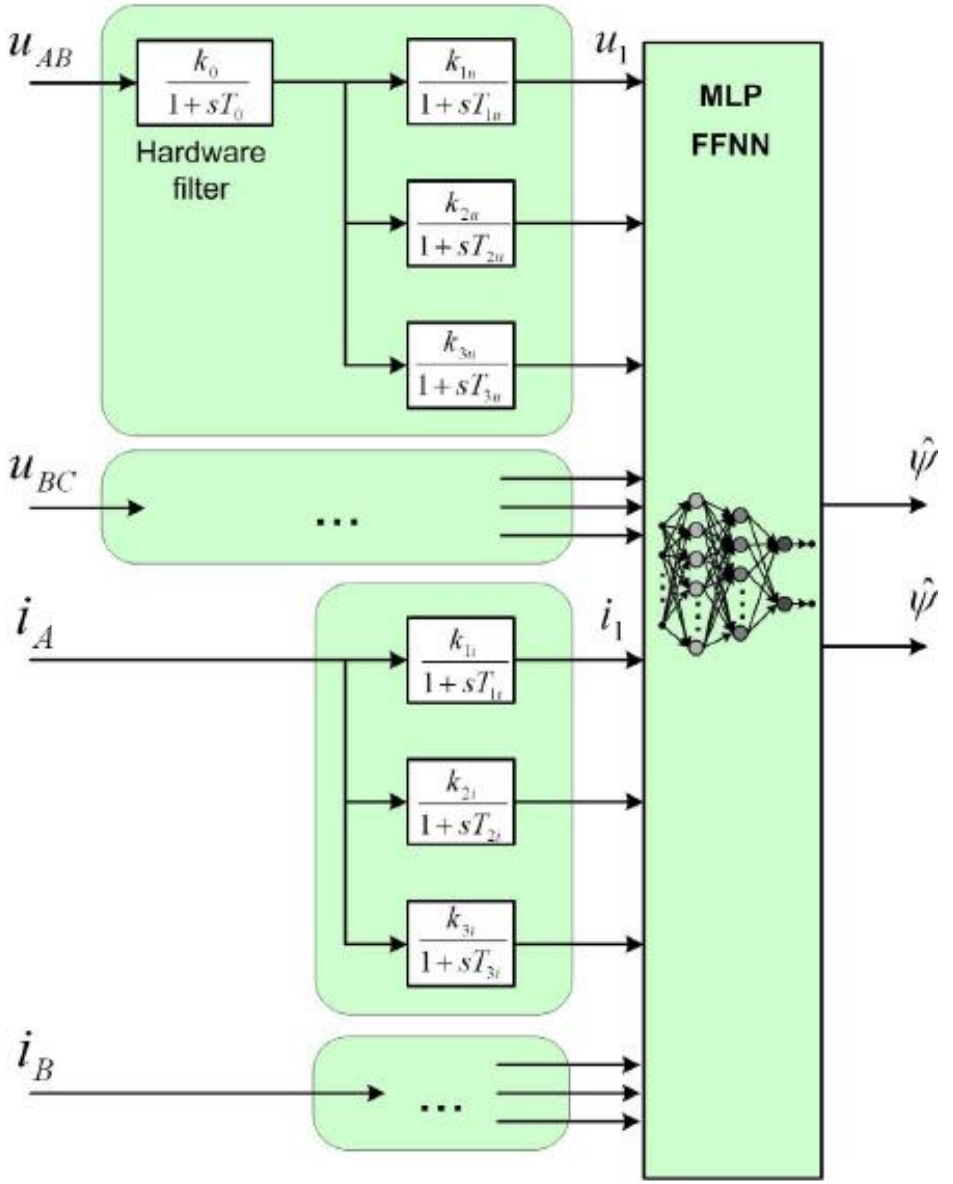

Fig. 8 Visual scheme of the fuzzy logic neuro net controller.

some degrees, so that the term crash is used as a cause of traffic accidents an image pick-up device is mounted at a front portion of an automobile to pick up ever-changing images as analog image data.

The speed sensor supplies velocity data to the signal processor through the input interface. The 
velocity data represent an actual velocity of the automobile at the time instant when the image pick-up device picks up an image of a view. Likewise, the steering gear ratio sensor supplies steering gear ratio data to the signal processor through the input interface. The signal processor comprises a CPU (central processing unit), a ROM (read-only memory) and a RAM (random-access memory). To evade potentially dangerous objects, CPU carries out calculation operation in response to the image, velocity and steering gear ratio data given through the input interface. CPU performs proper functions according to programs. Fig. 9 shows block scheme of predictive diagnostic control system. The output of the signal processor is transmitted through an output interface. ROM stores a table relating to numerical values required for the calculation. It also stores a table representing operational amount for a safety drive ensuring arrangement. On the other hand, RAM stores programs for use in calculating an optimum operational amount for the safety drive ensuring arrangement. Result of computer calculation presented in Fig. 10, a program for this purpose is disclosed in intelligent vehicle. The signal processor first determines,

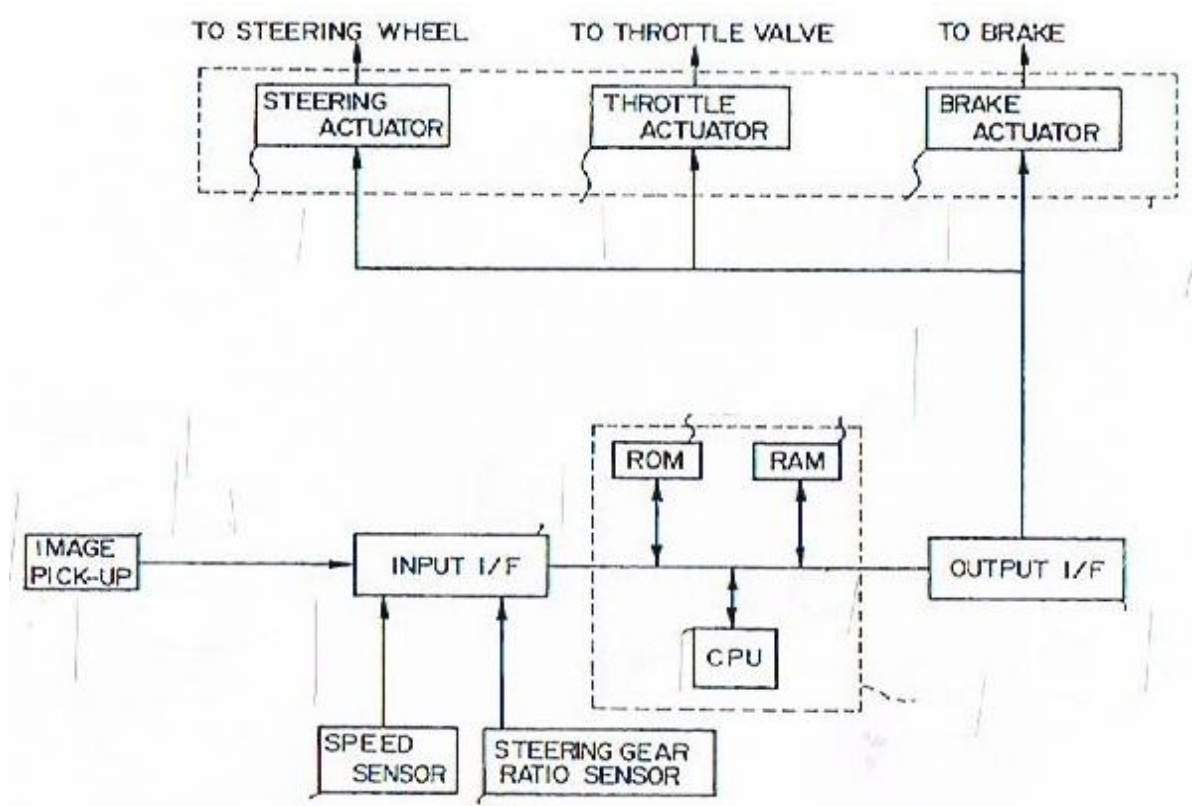

Fig. 9 Block scheme of predictive diagnostic control system [4].
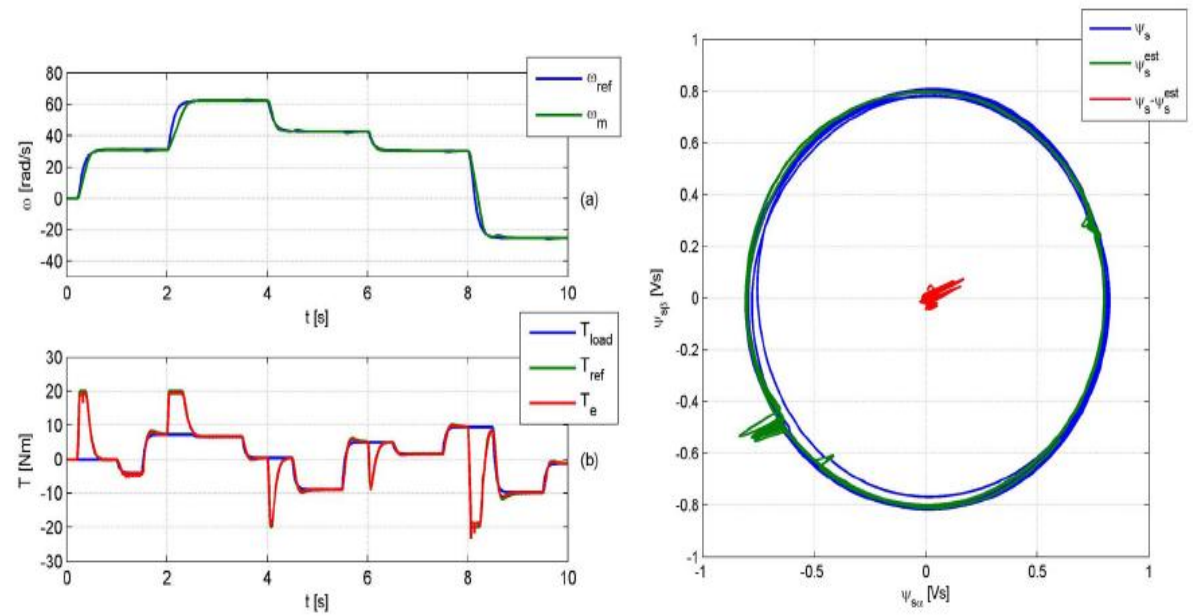

Fig. 10 Changes of rotational speed and driving torque at the correct work in traction motor. 
according to the picked up image data, whether there is a space available on the roadway to pass through. When there is enough space to pass through and a potentially dangerous object is present on the roadway, the signal processor calculates optimum operational amount for the safety drive for ensuring arrangement to operate the same.

The safety drive ensuring arrangement consists of a steering actuator, a throttle actuator and a brake actuator. If the signal processor determines that it is necessary to operate these actuators, it produces steering gear ratio command, set velocity command, and brake operation command. The steering actuator, the throttle actuator and the brake actuator are operated depending on the condition in response to the steering gear ratio command, the set velocity command and the brake operation command, respectively. The actuators for use are in actuating occupant protecting mechanism such as a brake device. The steering actuator is a hydraulic actuator for use in rotating steering wheel (not shown) in an emergency. In this event, the steering wheel is automatically rotated according to the steering gear ratio and rotational direction indicated by the steering gear ratio command. The operational amount of the steering or hydraulic actuator can be controlled in a well-known manner through a servo valve and a hydraulic pump, both of which are not shown in the figure. The throttle actuator acts to adjust opening amount of a throttle valve (not shown) to decrease speed while evading objects or so on. The brake actuator performs a function to gradually decrease speed of a vehicle in response to the brake operational command. The brake actuator is also capable of achieving sudden brake operation, if necessary. As mentioned above, CPU carries out its operation with tables and programs. This image pick-up device is any one of suitable devices such as a CCD (charge-coupled-device) camera. The analog image data obtained by the image pick-up device are supplied to the input interface [1, 4]. The input interface serves as an analog-to-digital converter for converting the analog image data into digital image data. More particularly, the picked up images are digitized by means of dividing the same into tiny pixels (data elements) isolated by grids. It is preferable to eliminate noises and distortions at this stage. The input interface is also connected to a speed sensor, a steering gear ratio sensor and a signal processor.

\section{Conclusion}

The control system of hybrid drive wheel vehicle should take into account: kind of the drive (internal-combustion drive, electric drive), hybrid drive, kinematic system of vehicle (kind of the ignition, system of the drive carriage), and exploitive (the ground configuration, maximum speeds and accelerations) parameters. The use of deck-computers in hybrid vehicle will assure a realization of composite algorithms of the control, aside from of the driver interference, economic works of the vehicle and safe the vehicle exploitation. Quasi-dynamic control algorithms, the peck of modern measuring-sensors and intelligent control systems helping the driver work assure safe and energy-saving during vehicle exploitation. Diagnostic system installed in the vehicle signal potential damage states and prevented to the vehicle damage.

\section{References}

[1] Szymański, Z. 2013. Quasi Dynamic Model of Traction Vehicle with Electric and Hybrid Drive System. Czasopismo Techniczne Politechniki Krakowskiej nr.5, Kraków r. (In Polish)

[2] Szymański, Z. 2007. Multilevel Control System of Traction Vehicle Drive System with Electric and Hybrid Drive. Czasopismo Techniczne Politechniki Krakowskiej nr.5, Kraków. (In Polish)

[3] Chee Shen Lim, Rahim, N. A., Wooi Ping Hew, and Levi, E. 2013. "Model Predictive Control of a Two-Motor Drive with Five-Leg-Inverter Supply." IEEE Trans. Ind. Electron 60 (1): 54-65.

[4] Tomoyoki, N. 1996. "Vehicle Crash Predictive and Evasive Operation System by Neural Network." United States Patent No. 5, 541, 590. 
[5] Miller, J. M., Gao, Y., and Ehsani, M. 2005. "Hybrid Electric Vehicle: Overview and State of Art." Proceedings of the IEEE ISIE'05, June, Dubrovnik, 307-15.

[6] "Engineering Smart Control System." Brochures of Siemens and ABB.

[7] Lucente, G., and Montari, M. 2005. "Hybrid Modeling of a Car Driveline for Servo-Actuated Gear Shift." Proceedings of the IEEE ISIE'05, June, Dubrovnik, 223-8.

[8] Szymański, Z. 2005. "Optimum Control System of Wheel and Rail Vehicle with Hybrid Drive System." Proceeding of International Conference MET'05, Październik, Warszawa. (Printed in Polish) 